\title{
Ultrasound Features of the Normal and Pathologic Periosteum
}

\author{
Antoine Moraux, MD, Salvatore Gitto, MD, Stefano Bianchi, MD
}

Received May 1, 2018, from the Imagerie Médicale Jacquemars Giélée Lille Ramsay Générale de Santé, Hôpital Privé La Louvière, Lille, France (A.M.); and Postgraduate School in Radiodiagnostics, Università Degli Studi di Milano, Milan, Italy (S.G.); Cabinet Imagerie Médicale, Geneva, Switzerland (S.B.). Manuscript accepted for publication July 2, 2018.

Address correspondence to Salvatore Gitto, MD, Postgraduate School in Radiodiagnostics, Università Degli Studi di Milano, Via Festa del Perdono 7, 20122 Milan, Italy.

E-mail:sal.gitto@gmail.com

Abbreviations

$M R$, magnetic resonance; US, ultrasound

doi: $10.1002 /$ jum.14762
Thickening and elevation of the periosteum from the underlying bone cortex, defined as a periosteal reaction, can be associated with several bone disorders. Although ultrasound (US) has limited possibilities in assessing bones, it can depict a periosteal reaction earlier than plain radiography, thus indicating underlying bone disorders. This pictorial essay aims to illustrate the normal and pathologic US appearances of the periosteum in both children and adults. Several disorders are discussed, such as pediatric bone trauma, infections and tumors, as well as trauma, overuse, including medial tibial stress syndrome, and finally certain seronegative spondyloarthropathies in adults. Whenever US depicts a periosteal reaction, a correlation with clinical and laboratory data is mandatory to differentiate different bone disorders. Computed tomography or magnetic resonance imaging must be performed when an infection or a tumor is suspected based on both US and the clinical presentation.

Key Words-bone; musculoskeletal imaging; periosteal reaction; periosteum; ultrasound

$\mathrm{I}$ $\mathrm{n}$ recent years, ultrasound (US) has progressively gained a central role in evaluations of the soft tissue structures of the 1 musculoskeletal system. The inability of US to penetrate cortical bone has, however, limited its usefulness in the assessment of bone and related disorders, and the periosteum itself is barely visible with US under healthy conditions. Several pathologic conditions affecting the bone may produce a periosteal reaction, which is a thickening and elevation of the periosteum from the underlying cortex. ${ }^{1}$ Different forms of periosteal reactions may result from various etiologies, including trauma, infections, tumors, and some metabolic and arthritic disorders. A periosteal reaction is conventionally assessed by plain radiography as a first-line imaging modality and computed tomography and magnetic resonance (MR) imaging as second-line modalities. Radiographic findings are positive only when the periosteum is calcified. In such cases, it presents as a thin linear calcification located at some distance from the bone surface or more irregular local calcifications depending on the causative disorder. ${ }^{1}$

Ultrasound is able to detect periosteal reactions earlier than plain radiography. ${ }^{2}$ Since US is often the first-line imaging modality in the evaluation of soft tissues of the extremities, a periosteal study must always be part of every musculoskeletal US examination. The US depiction of a pathologic periosteum together with no local tendon or joint abnormalities suggests a disorder of the underlying bone. A comparison with the healthy contralateral side is mandatory when subtle periosteal changes are present. Color 
Doppler US allows for an accurate assessment of local hyperemia. ${ }^{2}$ The purpose of this pictorial essay is to illustrate the normal and pathologic appearances of the periosteum on US imaging in both children and adults, with a focus on disorders in which US can play a role for diagnosis.

\section{Anatomy and Physiology of the Periosteum}

The periosteum is a membrane that covers nearly all bone structures with the exception of their intraarticular surfaces. It consists of an outer fibrous layer and an inner layer, also known as the cambium, which has considerable osteoblastic potential. The cambium and the periosteum as a whole are thick in children and become progressively thinner with age. In childhood, the periosteum is contiguous with a ring of perichondrium at the physeal level, until it transforms into the periosteum with physeal closure. The periosteum has a main role in bone growth and repair after injury and is a substantial contributor to the blood supply of bone and skeletal muscle. It also provides an attachment for muscles and tendons. ${ }^{3}$

\section{Ultrasound Technique and Normal Findings}

The US examination has to be bilateral and comparative. Either a linear or curvilinear transducer can be used according to the depth of the bone to be assessed. All structures from subcutaneous tissue to the cortex must be imaged with both grayscale and

Figure 1. Normal US appearance of the periosteum in a 24-yearold man. On a longitudinal sonogram, the periosteum (arrows) appears as a hypoechoic band overlying the anterior cortex of the tibia. It is thinner and less well shown by US than in children (see Figure 2). T indicates tibia.

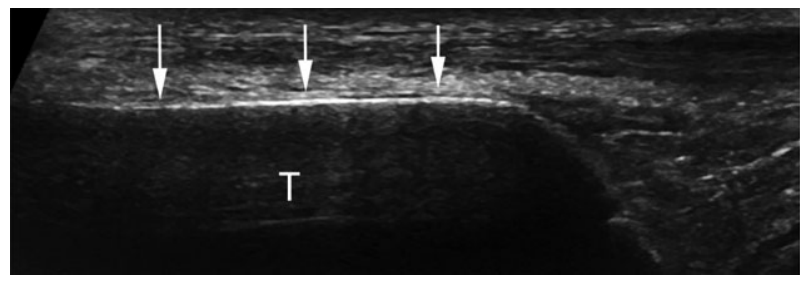

Doppler modes. On US imaging, healthy cortical bone appears as a regular, highly hyperechoic line with posterior acoustic shadowing and some reverberation artifacts. ${ }^{4}$ The normal periosteum is barely visible with US in healthy adults (Figure 1); however, it is clearly visualized as a thin hypoechoic band located over the bone cortex in children (Figure 2A). No Doppler signal is seen in the healthy periosteum, but the examination can detect the nutrient arteries perforating the periosteum and entering the bone cortex (Figure 2B). Under normal conditions, US is not able to depict structures that are deep to the bone cortex. ${ }^{2}$ Because many US features of disorders of the periosteum are unspecific, a US examination must be performed in light of clinical findings and a radiographic evaluation.

\section{Disorders of the Periosteum in Children}

\section{Pediatric Trauma}

In pediatric patients, the thick and strong periosteum often acts as a hinge that inhibits displacement in fractures. ${ }^{5}$ This action helps in remodeling the injured

Figure 2. Normal US appearance of the periosteum in a 5-year-old boy. A longitudinal sonogram (A) of the distal tibia depicts the periosteum (arrows), which appears as a thick hypoechoic band overlying the anterior cortex of the tibia. Note how the periosteum is contiguous with the perichondrium (arrowhead) at the physeal level, overlying the growth cartilage (asterisks). On a longitudinal Doppler image (B), a nutrient artery of the distal femur perforates the periosteum and enters the bone cortex. $\mathrm{F}$ indicates femur; and $\mathrm{T}$, tibia.

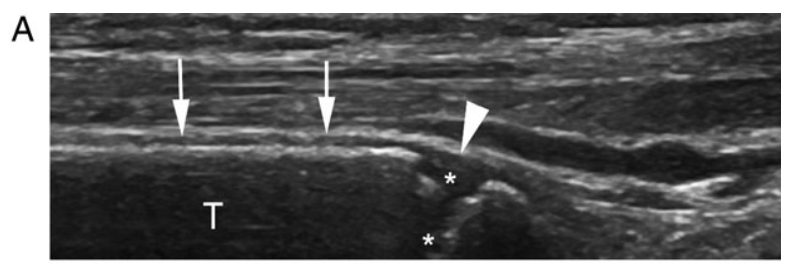

B

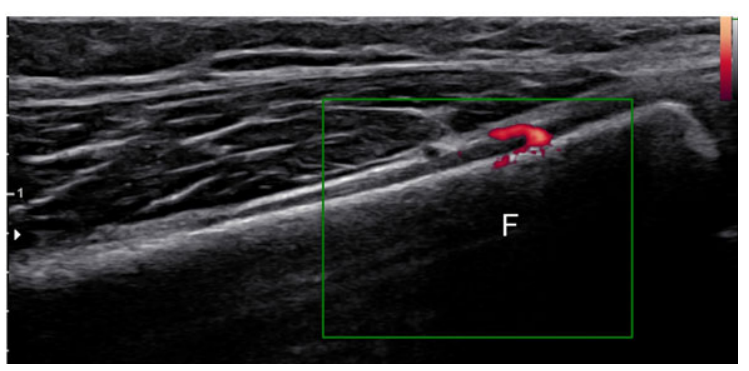


bones and allows for fast healing but may result in a missed diagnosis of a nondisplaced fracture on plain radiography. Ultrasound is helpful when radiographs are unremarkable, but a history of a recent trauma and local pain raise the suspicion of a fracture, such as a toddler ${ }^{6}$ or nondisplaced Salter-Harris fracture. ${ }^{7-9}$ The periosteal reaction consists of a subperiosteal hemorrhage that results from traumatic detachment of the periosteum and related injury to nutrient vessels. Ultrasound findings of pediatric fractures (Figure 3) include the following: (1) soft tissue

Figure 3. Hairline fracture of the femur in a 7-year-old boy. On an anteroposterior radiograph (A), a fracture line (arrows) is barely visible in the left femur. On a sonogram (B), a subperiosteal hematoma (arrows) and the fracture line (arrowhead) are shown. F indicates femur; and Q, quadriceps muscle.

A

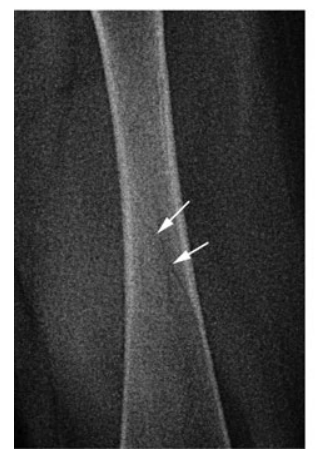

B

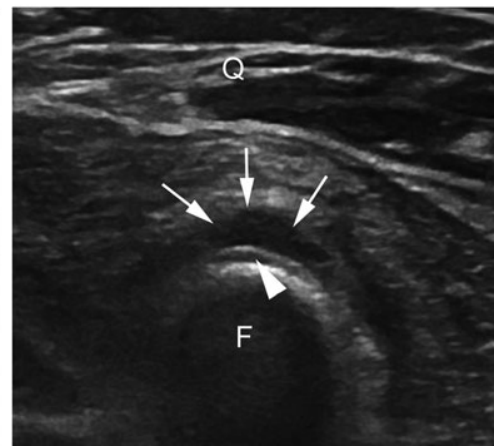

swelling and hyperemia reflecting local inflammation; (2) hypoechoic thickening of the periosteum/physeal perichondral ring, which is crescent shaped on axial images and suggestive of subperiosteal hematoma; and (3) cortical bone interruption. Additional findings of Salter-Harris fractures (Figure 4) are physeal widening and bone fragmentation at the physis. ${ }^{10-13}$ A comparison with the healthy contralateral side often helps to distinguish the normal periosteum and growth cartilage from injured ones.

\section{Pediatric Bone Infections}

The periosteum plays a central role in the pathogenesis of acute osteomyelitis. The subperiosteal space and periosteum are affected as a result of microorganism spread from the medullary cavity through the cortical bone canals, such as the Haversian and Volkmann canals. ${ }^{14,15}$ Some authors also referred to the osteoperiosteal area as one main focus of infection and adopted the term "acute osteoperiostitis." 16 Osteomyelitis can cause a localized periosteal reaction anywhere but primarily in long bones, which are commonly affected in children. ${ }^{1}$ The clinical presentation includes local pain, skin redness, weakness, and fever. Ultrasound is able to identify periosteal changes associated with osteomyelitis even several days before any apparent finding on plain radiography. ${ }^{17}$ The US examination of the affected bone must be circumferential and comparative.

Figure 4. Type 1 Salter-Harris fracture of the fibula in an 8-year-old boy. An anteroposterior radiograph (A) shows a nondisplaced type 1 Salter-Harris fracture of the distal fibula associated with small bone avulsion fragments (arrowheads) and swelling of the adjacent soft tissues (asterisk). The corresponding sonogram (B) shows widening of the growth cartilage (arrows), a subperiosteal hematoma (arrowheads), and soft tissue local edema (asterisk). The normal appearance of the periosteum and growth cartilage is shown on the contralateral side $(\mathbf{C})$.

A

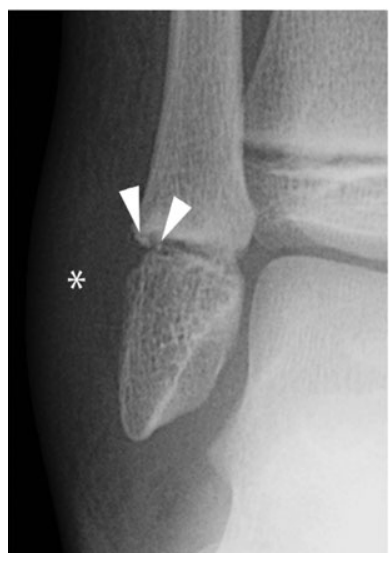

B

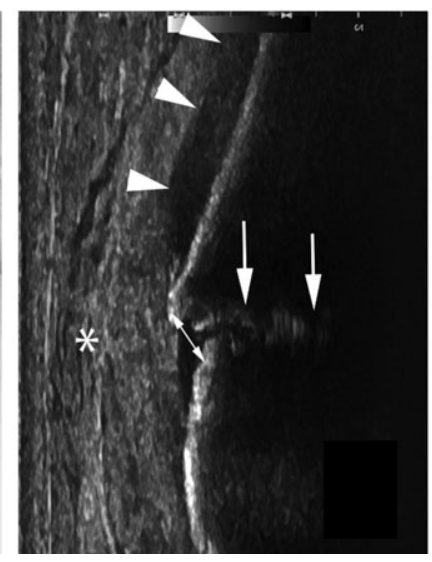

C

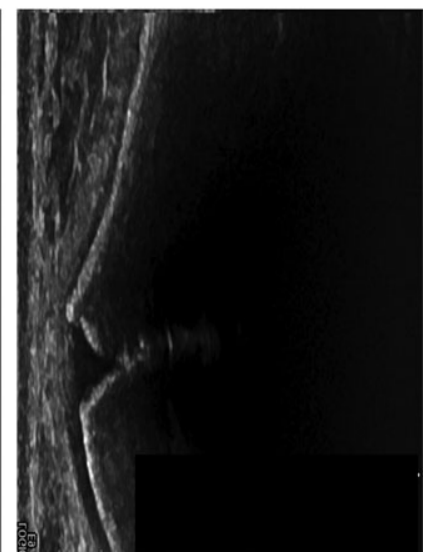


Ultrasound findings of acute osteomyelitis (Figures 5 and 6) are as follows: (1) hyperechoic edematous infiltration of soft tissues, which is the earliest sign but is nonspecific; (2) thickening and elevation of the periosteum by a thin layer $(<3 \mathrm{~mm})$ of hypoechoic subperiosteal fluid, which represents an early subperiosteal collection; (3) a larger fusiform subperiosteal collection, which is suggestive of a subperiosteal abscess and shows variable echogenicity based on the pus consistency; and (4) hyperemia within and around the periosteum and in the surrounding soft tissues on Doppler imaging. Early detection of the subperiosteal collection is crucial because US-guided aspiration or surgical drainage has to be considered in such cases. ${ }^{18-21}$ Later, US can further depict the following: (5) subtle

Figure 5. Acute osteomyelitis of the tibia in an infant. A longitudinal sonogram obtained over the tibia shows an abscess (asterisks) located below the periosteum (arrows). $T$ indicates tibia.

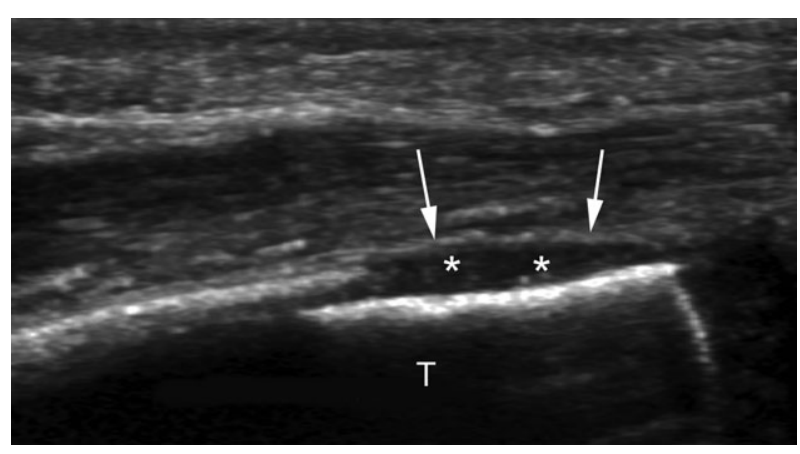

Figure 6. Acute osteomyelitis of the tibia in an infant. A longitudinal power Doppler image obtained over the tibia shows a subperiosteal abscess (asterisk) and hypervascular changes of the periosteum (arrows). T indicates tibia. Courtesy of Nathalie Boutry MD PhD Department of Paediatric Imaging, Hospital Jeanne de Flandre, CHU Lille, France.

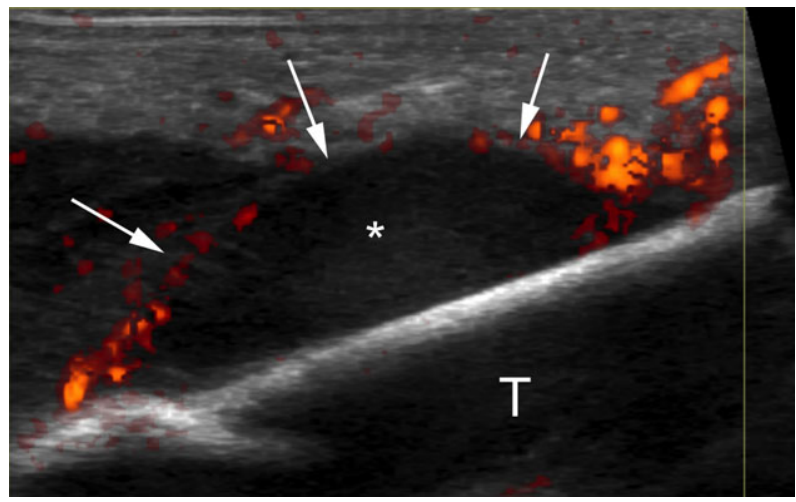

permeative changes of the cortical bone, which is intact but less reflective than the healthy cortex; and (6) a soft tissue abscess that appears as a hypoechoic or anechoic fluid collection and may extend around the bone surface. Intra-articular effusion and synovial thickening of the adjacent joint can be identified in cases of concomitant septic arthritis. In chronic osteomyelitis, US may reveal cortical defects, fistulas, and sequestrations. $^{22}$

\section{Pediatric Bone Tumors}

The main role of US in the diagnosis of bone tumors is for the guidance of percutaneous needle biopsies. ${ }^{23}$ Pediatric bone tumors are discussed here because aggressive tumors can mimic certain bone disorders, such as acute osteomyelitis. Aggressive malignant or benign bone tumors become accessible to US assessments after they penetrate the cortex and produce a periosteal reaction or an extraosseous mass. ${ }^{24}$ Most malignant bone tumors diagnosed in children and young adults are osteosarcoma and Ewing sarcoma. Leukemia and lymphoma as well as subperiosteal osteoid osteoma can also produce extensive periosteal reactions in children and young adults. ${ }^{1}$ The clinical presentation of bone tumors is often subtle, and pain is the most common symptom in early stages. On US imaging (Figures 7 and 8), (1) the periosteal reaction

Figure 7. Ewing sarcoma of the clavicle. A plain radiograph (A) shows irregular periosteal apposition (arrowheads). The corresponding sonogram (B) depicts cortical bone irregularities (arrowheads) and a spiculated periosteal reaction (arrows) with a sunburst appearance. $\mathrm{C}$ indicates clavicle.

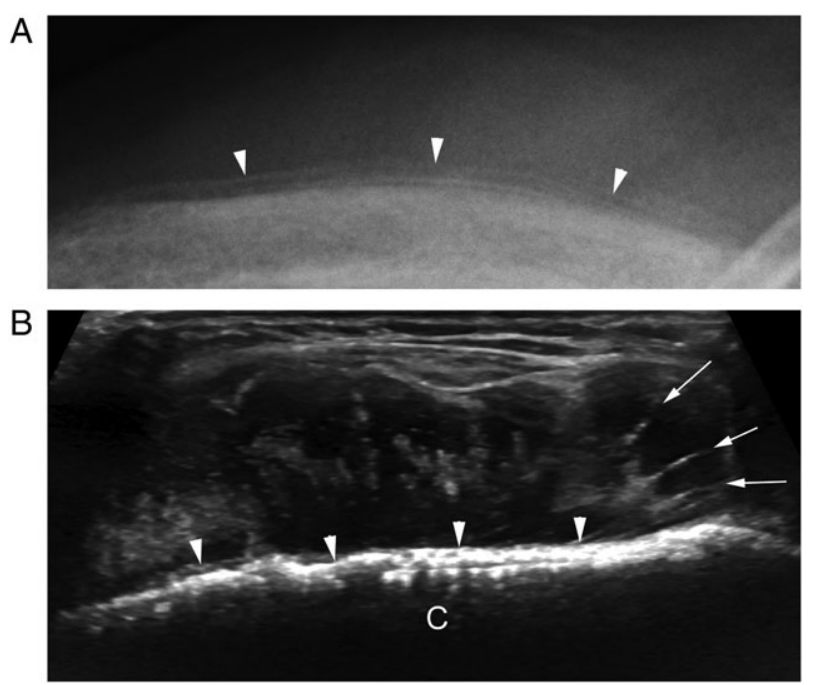


can be either nonossified, and it appears as a thin hyperechoic raised line, or mineralized, and it appears as a smooth continuation of the cortex; and (2) periosteal hyperemia may be seen on Doppler imaging. Additional findings of aggressive bone tumors are as follows: (3) discontinuity or irregularity of the cortex, reflecting cortical erosion and destruction; (4) an extraosseous tumor component, which shows a variable appearance based on the degree of ossification and necrosis; and (5) displacement or encasement of an adjacent neurovascular bundle. Additionally, if a pathologic fracture occurs, it may be identified as a focal interruption and a step in the hyperechoic cortex. $^{24}$

\section{Disorders of the Periosteum in Adults}

\section{Adult Trauma and Overuse}

Nondisplaced, radiographically undetected acute fractures show a periosteal reaction and can be assessed with US. The US examination must always be focused on the clinical findings: ie, over the most painful area. During the US examination, pain is typically elicited with sonopalpation over the suspected site of injury. Early US findings (Figures 9 and 10) are as follows: (1) soft tissue swelling, reflecting local edema; (2) periosteal hypoechoic thickening; (3) periosteal hyperemia on Doppler imaging; and (4) an interruption of the bone surface. ${ }^{25,26}$ In cases of trabecular fractures (Figure 11), periosteal hypoechoic thickening but no discontinuity of the bone can be seen. In
Figure 9. Thumb radial sesamoid fracture in a 52-year-old man with a previous fall. Plain radiography (not shown) was unremarkable. An axial sonogram (A) of the injured thumb shows periosteal thickening (black arrowhead) of the radial sesamoid bone and a fracture (white arrow) that splits the sesamoid bone in two fragments (asterisks). The normal sesamoid (Ses) of the contralateral thumb is shown for comparison, and the healthy periosteum (white arrowhead) is very thin and overlies the cortex (B). Black arrows indicate the flexor pollicis longus tendon.

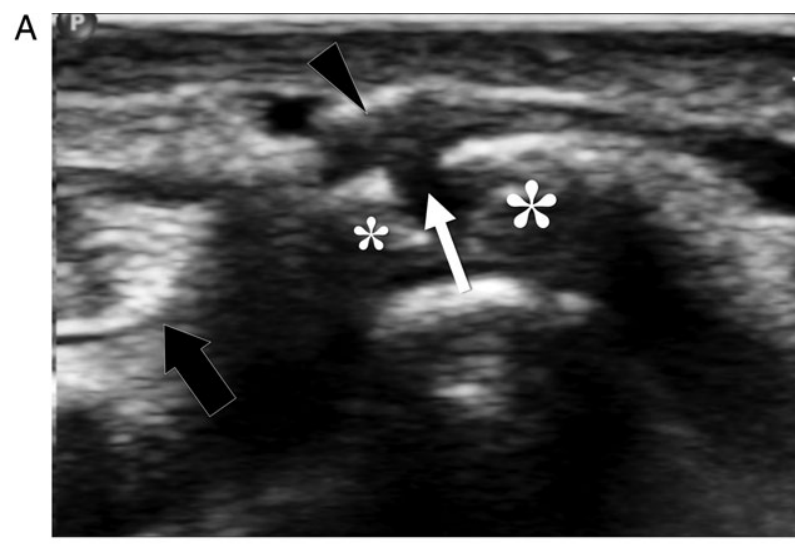

B

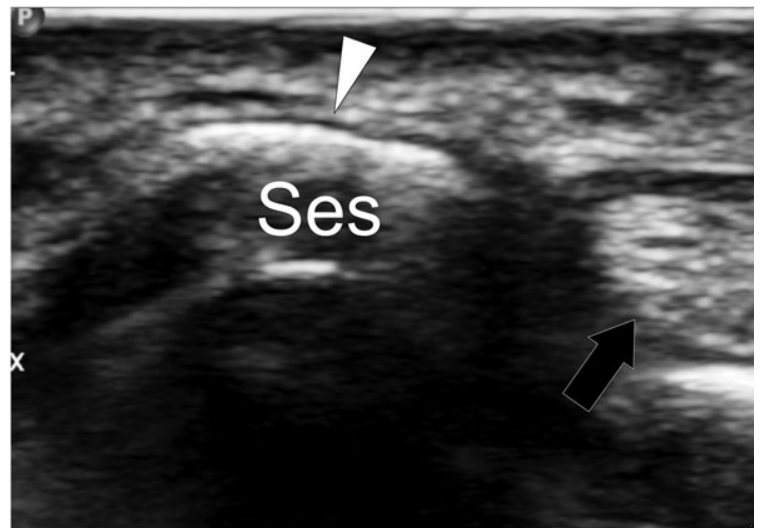

Figure 8. Osteoid osteoma of the third cuneiform in a 21-year-old man with tarsal pain during sport activities and at night. Plain foot radiography (not shown) was unremarkable. An axial sonogram (A) shows periosteal thickening (arrowheads) overlying the slightly irregular cortex of the third cuneiform. On a sagittal color Doppler image (B), hyperemia of the periosteum (arrowheads) is shown. Computed tomography (C) depicts an osteoid osteoma with a calcified nidus (arrow) of the third cuneiform. The osteoid osteoma was proven at surgery. CN3 indicates third cuneiform.

A

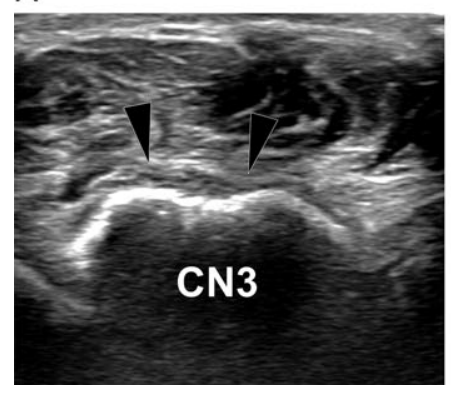

B

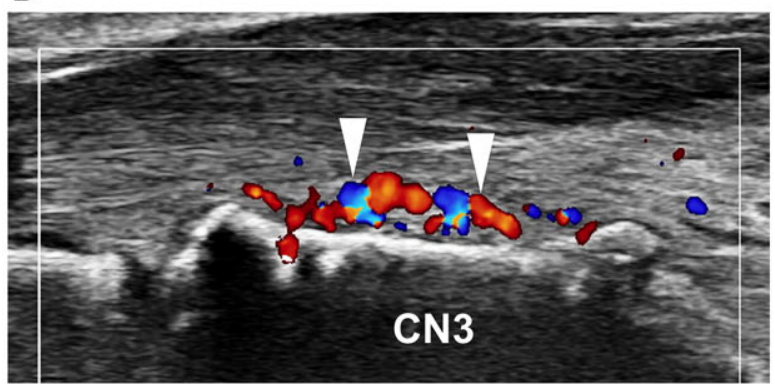

C

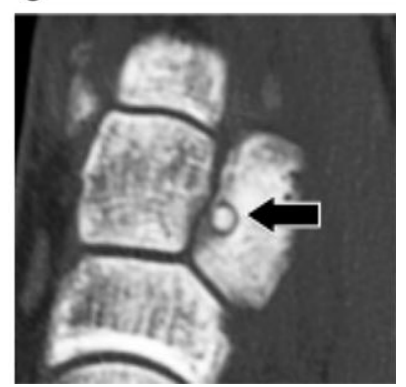


Figure 10. Humeral greater tuberosity fracture in a 47-year-old man with a previous fall. Plain radiography (not shown) was unremarkable. A coronal oblique sonogram obtained over the lateral aspect of the proximal humerus shows a focal interruption (arrow) in the bone cortex that is consistent with a nondisplaced fracture. Note local thickening of the periosteum (arrowheads).

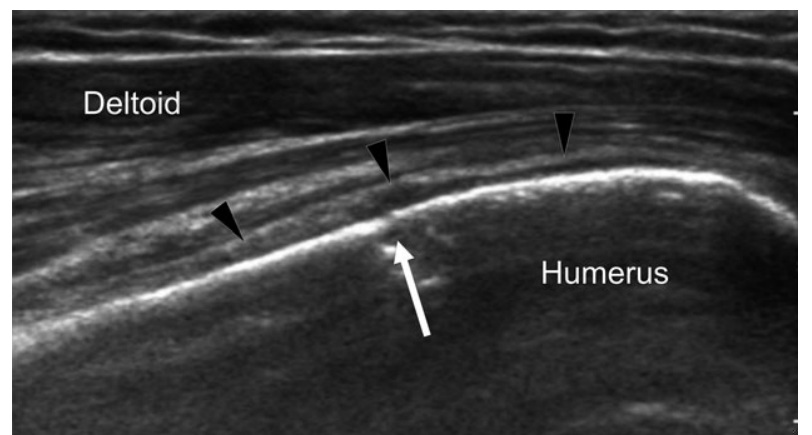

subacute and chronic stages, US depicts bone callus formation and may reveal a fracture nonunion by showing active and passive motion of the bone fragments at the fracture site under dynamic assessment. ${ }^{2}$

Stress fractures are secondary to repeated overuse and overload. They can be classified as fatigue fractures, which result from excessive stress on healthy bones and are encountered in both recreational and professional athletes; and insufficiency fractures, which result from normal stress on abnormally weak bones due to systemic disorders, such as osteoporosis and rheumatoid arthritis. Stress fractures are more common in the lower extremities than in the upper extremities, and they mostly involve the tibia, tarsals, metatarsals, and femur. ${ }^{27}$ The clinical presentation may include a recent history of increased intensity and duration of physical activity and local pain that is

Figure 11. Trabecular fracture of the fibula. Coronal oblique grayscale (A) and axial color Doppler (B) sonograms show periosteal thickening (arrows) and adjacent soft tissue edema (asterisks). Periosteal hyperemia (arrowhead) is shown on Doppler imaging. No cortical interruption is shown. On coronal oblique T1-weighted $(\mathbf{C})$ and fat-suppressed proton density-weighted (D) MR images, a trabecular fracture line (arrowheads) associated with bone marrow edema and periosteal reaction (arrows) are depicted. F indicates fibula.

A

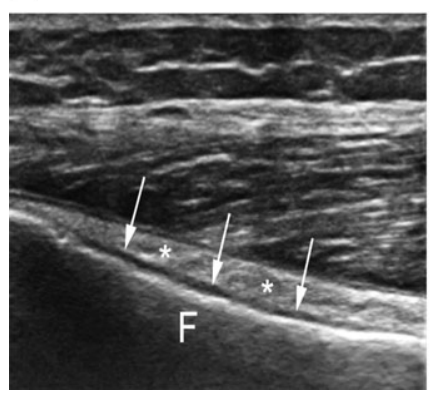

B

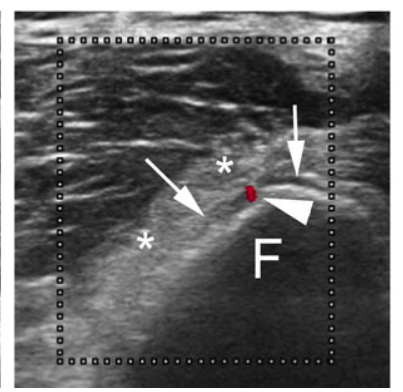

C

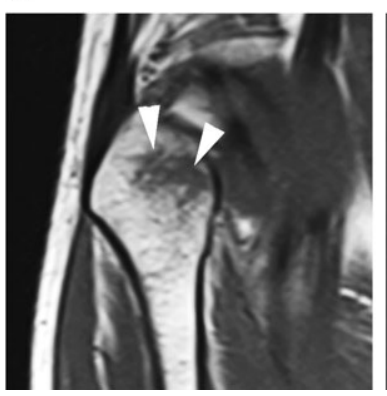

D

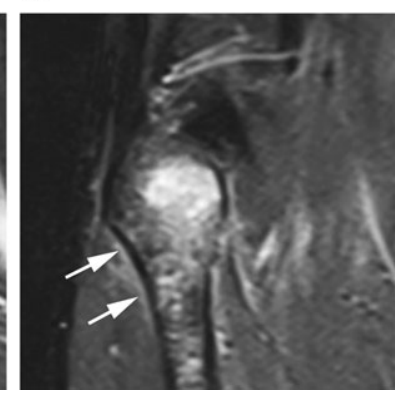

Figure 12. Tibial stress fracture in a 28-year-old runner. On longitudinal (A) and axial (B) sonograms, the periosteum is thick (white arrowheads) and shows focal calcifications (black arrowheads). Note focal irregularity of the bone cortex and hyperechoic edema of the deep soft tissues (white arrows). Axial T1-weighted (C) and T2-weighted (D) MR images confirm these findings and also show bone marrow edema (asterisks) and a fracture line (black arrow).

A

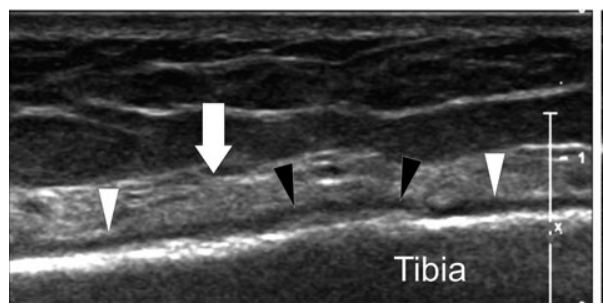

B

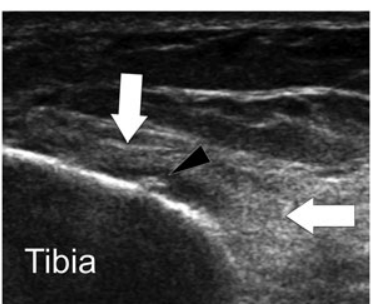

C

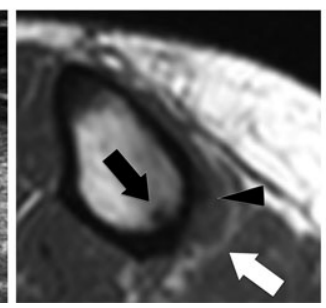

D

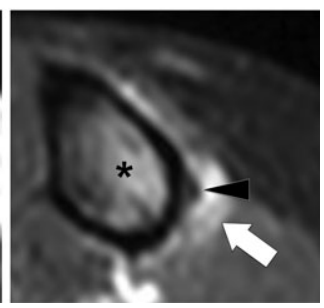


exacerbated by exercise and diminished by rest. Early US findings (Figures 11-15) at the site of fracture include the following: (1) hyperechoic soft tissue swelling that reflects edema; (2) hypoechoic periosteal thickening that overlies the hyperechoic bone cortex; (3) posterior shadowing related to periosteal thickening, which is only seen in small bones such as the metatarsals; and (4) an increased periosteal Doppler signal, reflecting local hyperemia. Late US findings (Figure 16) are irregularity or discontinuity of the bone cortex and calcified bone callus formation. ${ }^{28-33}$

\section{Medial Tibial Stress Syndrome}

Medial tibial stress syndrome, also known as shin splints, is a common cause of exercise-induced pain along the middle to distal posteromedial aspect of the tibia and has an unclear origin. ${ }^{34}$ It was originally related to an inflammatory periostitis due to excessive traction by the tibialis posterior and soleus muscles. More recent studies have hypothesized that local overload results in microdamage within the bone that normally leads to bony adaptation processes but, over a certain threshold, cannot be repaired. ${ }^{35}$ Ultrasound features of medial tibial stress syndrome (Figure 17) include the following: (1) hyperechoic soft tissue swelling; (2) periosteal hypoechoic thickening on the posteromedial aspect of the tibia; and (3) permeative changes of the cortical bone, which is less reflective than the healthy cortex, probably because of cortical reabsorption. These findings are, however, nonspecific, and some authors have reported that they can also be found in athletes with no medial tibial stress syndrome. ${ }^{36}$

\section{Seronegative Spondyloarthropathies}

A periosteal reaction develops in seronegative spondyloarthropathies, such as psoriatic arthritis and reactive arthritis. In particular, bone proliferation is a key feature of psoriatic arthritis, and inflammatory periostitis may occur along the phalangeal shafts. ${ }^{1}$ Cutaneous psoriasis often precedes arthritis, which mainly involves large joints, such as the sacroiliac joints, and the toes and fingers with distal predominance. In the fingers, extrasynovial US findings of psoriatic arthritis (Figure 18) include the following: (1) soft tissue thickening, either diffuse or localized, and hyperemia on Doppler imaging; (2) a juxta-articular periosteal

Figure 14. Third metatarsal stress fracture in a 25-year-old runner. Grayscale (A) and color Doppler (B) longitudinal sonograms show focal thickening of the periosteum (black arrowheads). Periosteal hyperemia (open arrowheads) is shown on Doppler imaging. Note the normal periosteum more proximally (white arrowhead). The bone cortex is normal. The adjacent soft tissues are hyperechoic because of edema (white arrows).

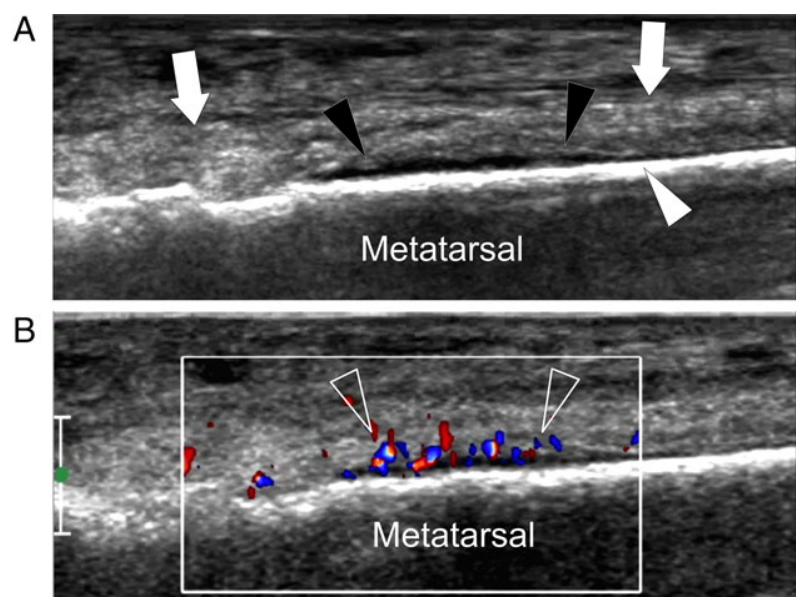

Figure 13. Calcaneal insufficiency fracture in an 82-year-old woman. Grayscale (A) and color Doppler (B) axial sonograms show periosteal thickening (arrowheads), cortical irregularities, and edema of the adjacent soft tissues. Periosteal and soft tissue hyperemia is shown on Doppler imaging. A contrast-enhanced T1-weighted MR image $(\mathbf{C})$ confirms these findings and shows bone marrow edema (asterisk) and a vertical fracture line (arrow).

A

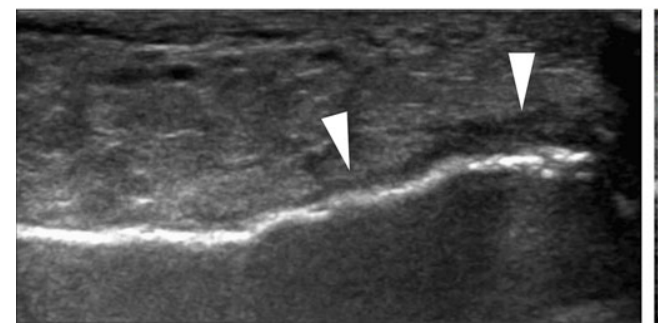

$\mathrm{B}$

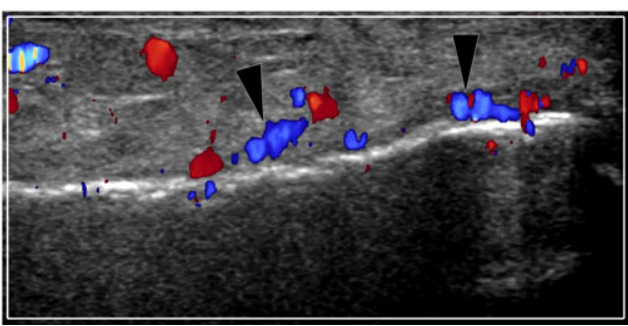

C

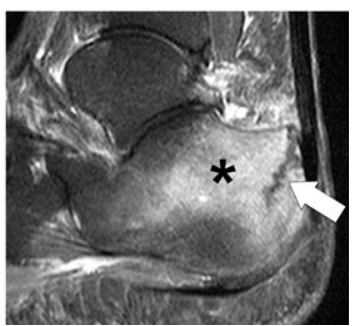


reaction, which appears as a thin hyperechoic raised line; (3) fine bone spicules at the capsule attachment site; and (4) enthesopathy of the deep flexor tendon at the base of the distal phalanx, which appears as hypoechogenicity of the distal tendon and cortical haziness reflecting bone remodeling. Additionally, synovitis, joint erosion, and tenosynovitis are common US findings. ${ }^{37-39}$

\section{Adult Bone Tumors}

Bone tumors frequently found in adults and the elderly include chondrosarcoma, myeloma, and

Figure 15. Tibial subchondral insufficiency fracture in a 77-year-old woman. Color Doppler coronal (A) and axial (B) sonograms show periosteal thickening (arrowheads) and hyperemia. The cortex is normal. A sagittal contrast-enhanced T1-weighted MR image (C) depicts a hypointense subchondral line suggestive of subchondral insufficiency fracture (arrow) and bone marrow edema (asterisk).
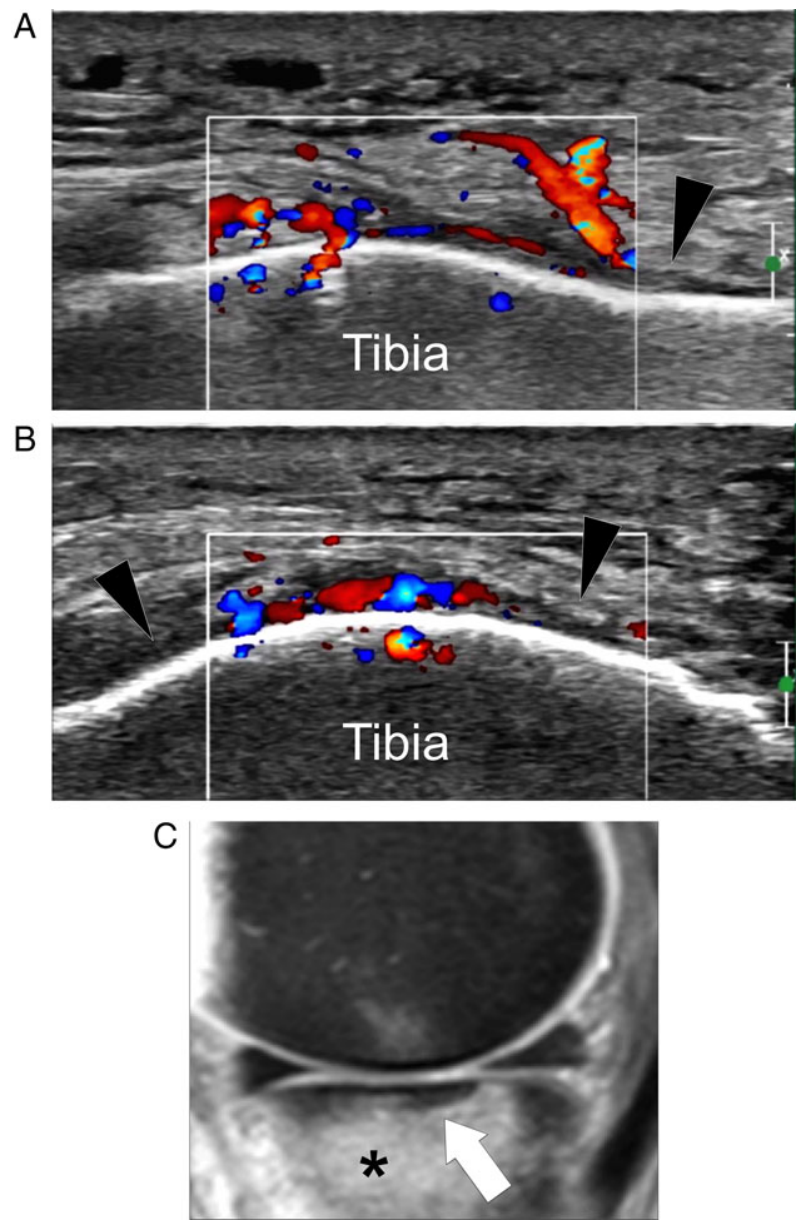

Figure 16. Untreated third metatarsal stress fracture in a 28-yearold avid runner. Grayscale (A) and color Doppler (B) longitudinal sonograms show well-evident thickening of the periosteum (black arrowheads) associated with local callus formation (black arrow) and adjacent soft tissue edema (white arrow). Hypervascular changes of the periosteum, adjacent soft tissues, and bone cortex (white arrowheads) are shown on Doppler imaging.

A

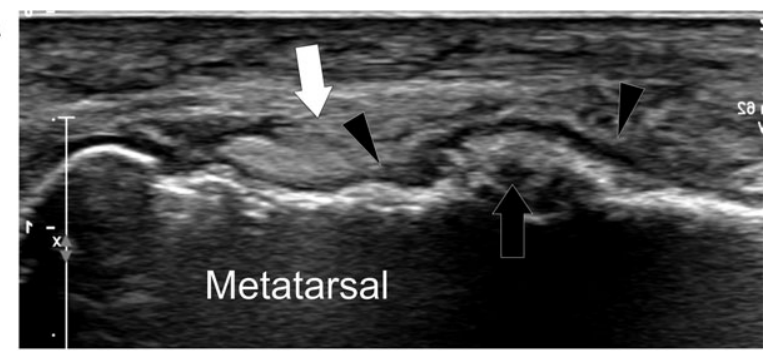

B

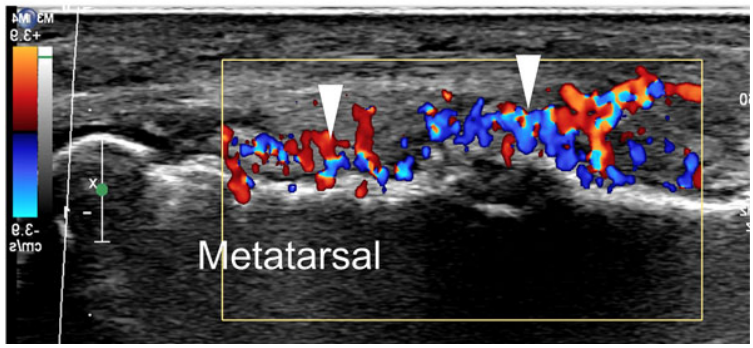

metastases. They may produce a periosteal reaction or an extraosseous mass and resemble the US appearance of pediatric bone tumors. However, the role of US in the diagnosis of adult bone tumors is limited to the guidance of percutaneous needle biopsies. ${ }^{24}$

\section{Conclusions}

A periosteal reaction can be associated with a variety of underlying bone disorders. Although US has limited value in assessing bones, it may depict a periosteal reaction as hypoechoic thickening and hyperemia of the periosteum earlier than plain radiography. Detection of a periosteal reaction with US, associated with a normal appearance of other musculoskeletal structures, directs the attention to a possible bone disorder. Nevertheless, as US findings are often nonspecific, correlation with clinical and laboratory data is necessary. We suggest that a careful US assessment of the periosteum must be a part of every US examination of the extremities. The US detection of a periosteal reaction must 
Figure 17. Medial tibial stress syndrome in a 24-year-old avid runner. An axial sonogram obtained over the junction between the middle and distal third of the right tibia (A) depicts focal thickening of the periosteum (white arrowhead), associated with marked edema of the adjacent soft tissues (black arrowheads). Note the normal appearance of the superficial soft tissues. The tibial cortex is normal. The normal appearance of the periosteum and local soft tissues is shown on the contralateral side for comparison (B).

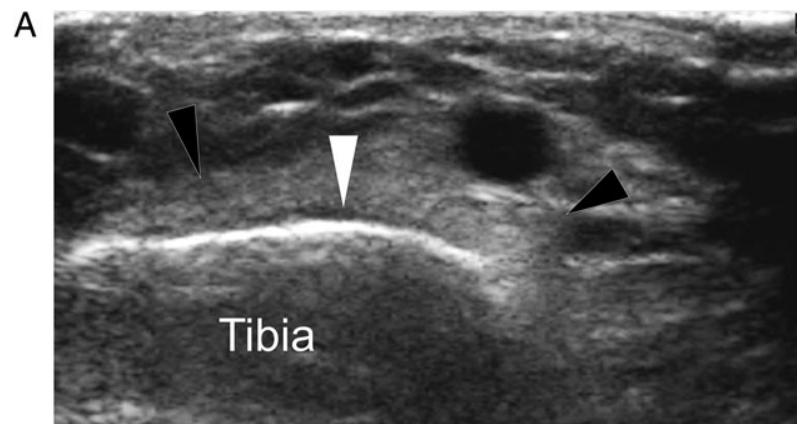

B

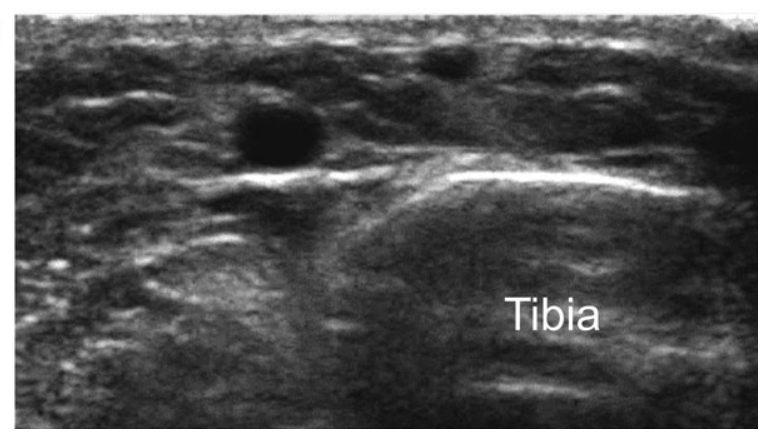

Figure 18. Psoriatic arthritis. A Doppler image shows thickening and hyperemia of the periosteum of the middle phalanx of the index finger, as well as thickening and hyperemia of the adjacent soft tissues.

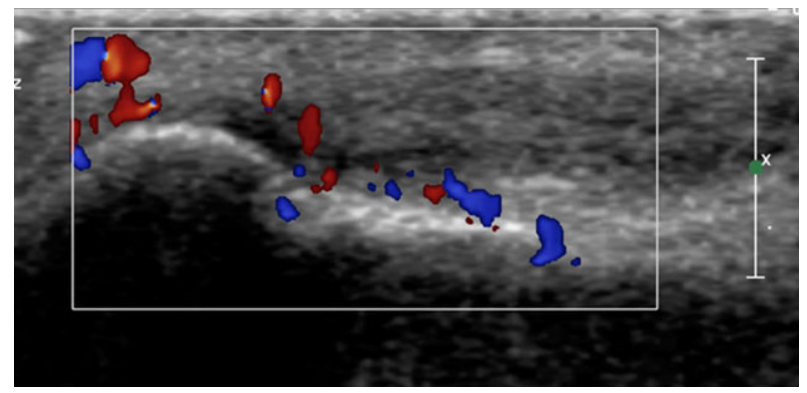

induce the sonologist to use a second-line imaging modality, such as computed tomography or MR imaging, when an infection or a tumor is suspected on the basis of both US findings and the clinical presentation.

\section{References}

1. Rana RS, Wu JS, Eisenberg RL. Periosteal reaction. AJR Am J Roentgenol 2009; 193:W259-W272.

2. Hoffman DF, Adams E, Bianchi S. Ultrasonography of fractures in sports medicine. Br J Sports Med 2015; 49:152-160.

3. Dwek JR. The periosteum: what is it, where is it, and what mimics it in its absence? Skeletal Radiol 2010; 39:319-323.

4. Erickson SJ. High-resolution imaging of the musculoskeletal system. Radiology 1997; 205:593-618.

5. Kao SC, Smith WL. Skeletal injuries in the pediatric patient. Radiol Clin North Am 1997; 35:727-746.

6. Lewis D, Logan P. Sonographic diagnosis of toddler's fracture in the emergency department. J Clin Ultrasound 2006; 34: 190-194.

7. Farley FA, Kuhns L, Jacobson JA, DiPietro M. Ultrasound examination of ankle injuries in children. J Pediatr Orthop 2001; 21:604-607.

8. Gleeson AP, Stuart MJ, Wilson B, Phillips B. Ultrasound assessment and conservative management of inversion injuries of the ankle in children: plaster of Paris versus Tubigrip. J Bone Joint Surg Br 1996; 78:484-487.

9. Supakul N, Hicks RA, Caltoum CB, Karmazyn B. Distal humeral epiphyseal separation in young children: an often-missed fracture-radiographic signs and ultrasound confirmatory diagnosis. AJR Am J Roentgenol 2015; 204:W192-W198.

10. Cho KH, Lee SM, Lee YH, Suh KJ. Ultrasound diagnosis of either an occult or missed fracture of an extremity in pediatric-aged children. Korean J Radiol 2010; 11:84-94.

11. Hubner U, Schlicht W, Outzen S, Barthel M, Halsband H. Ultrasound in the diagnosis of fractures in children. J Bone Joint Surg Br 2000; 82:1170-1173.

12. Simanovsky N, Hiller N, Leibner E, Simanovsky N. Sonographic detection of radiographically occult fractures in pediatric ankle injuries. Pediatr Radiol 2005; 35:1062-1065.

13. Simanovky N, Lamdan R, Hiller N, Simanovsky N. Sonographic detection of radiographically occult fractures in pediatric ankle and wrist injuries. J Pediatr Orthop 2009; 29:142-145.

14. Jaramillo D, Dormans JP, Delgado J, Laor T, St Geme JW III. Hematogenous Osteomyelitis in infants and children: imaging of a changing disease. Radiology 2017; 283:629-643.

15. Pineda C, Espinosa R, Pena A. Radiographic imaging in osteomyelitis: the role of plain radiography, computed tomography, ultrasonography, magnetic resonance imaging, and scintigraphy. Semin Plast Surg 2009; 23:80-89.

16. Labbe JL, Peres O, Leclair O, et al. Acute osteomyelitis in children: the pathogenesis revisited? Orthop Traumatol Surg Res 2010; 96:268-275.

17. Kaiser S, Rosenborg M. Early detection of subperiosteal abscesses by ultrasonography: a means for further successful treatment in pediatric osteomyelitis. Pediatr Radiol 1994; 24:336-339. 
18. Azam Q, Ahmad I, Abbas M, Syed A, Haque F. Ultrasound and colour Doppler sonography in acute osteomyelitis in children. Acta Orthop Belg 2005; 71:590-596.

19. Chau CL, Griffith JF. Musculoskeletal infections: ultrasound appearances. Clin Radiol 2005; 60:149-159.

20. Hammou A, Ben Chehida F, Bellagha I, Essaddem H, Gharbi HA. L'Échographie dans les infections osseuses. J Echogr Med Ultrason 1997; 18:221.

21. Riebel TW, Nasir R, Nazarenko O. The value of sonography in the detection of osteomyelitis. Pediatr Radiol 1996; 26:291-297.

22. Robben SG. Ultrasonography of musculoskeletal infections in children. Eur Radiol 2004; 14(suppl 4):L65-L77.

23. Wenaden AE, Szyszko TA, Saifuddin A. Imaging of periosteal reactions associated with focal lesions of bone. Clin Radiol 2005; 60:439-456.

24. Saifuddin A, Burnett SJ, Mitchell R. Pictorial review: ultrasonography of primary bone tumours. Clin Radiol 1998; 53:239-246.

25. Botchu R, Lee KJ, Bianchi S. Radiographically undetected coracoid fractures diagnosed by sonography: report of seven cases. Skeletal Radiol 2012; 41:693-698.

26. Celi J, de Gautard G, Della Santa JD, Bianchi S. Sonographic diagnosis of a radiographically undiagnosed hook of the hamate fracture. J Ultrasound Med 2008; 27:1235-1239.

27. Matheson GO, Clement DB, McKenzie DC, Taunton JE, Lloyd-Smith DR, MacIntyre JG. Stress fractures in athletes: a study of 320 cases. Am J Sports Med 1987; 15:46-58.

28. Arni D, Lambert V, Delmi M, Bianchi S. Insufficiency fracture of the calcaneum: sonographic findings. J Clin Ultrasound 2009; 37:424-427.

29. Bianchi S, Luong DH. Stress fractures of the ankle malleoli diagnosed by ultrasound: a report of six cases. Skeletal Radiol 2014; 43:813-818.
30. Bianchi S, Luong DH. Stress fractures of the calcaneus diagnosed by sonography: report of 8 cases. J Ultrasound Med 2018; 37: 521-529.

31. Bodner G, Stockl B, Fierlinger A, Schocke M, Bernathova M. Sonographic findings in stress fractures of the lower limb: preliminary findings. Eur Radiol 2005; 15:356-359.

32. Bousson V, Wybier M, Petrover D, et al. Stress fractures [in French]. J Radiol 2011; 92:188-207.

33. Khy V, Wyssa B, Bianchi S. Bilateral stress fracture of the tibia diagnosed by ultrasound: a case report. J Ultrasound 2012; 15: 130-134.

34. Reshef N, Guelich DR. Medial tibial stress syndrome. Clin Sports Med 2012; 31:273-290.

35. Burrus MT, Werner BC, Starman JS, et al. Chronic leg pain in athletes. Am J Sports Med 2015; 43:1538-1547.

36. Winters M, Bon P, Bijvoet S, Bakker EW, Moen MH. Are ultrasonographic findings like periosteal and tendinous edema associated with medial tibial stress syndrome? A case-control study. J Sci Med Sport 2017; 20:128-133.

37. Denis A, Lapegue F, Sans N, et al. Cross-sectional imaging of peripheral involvement in psoriatic arthritis [in French]. J Radiol 2010; 91:126-139.

38. Fournie B, Margarit-Coll N, Champetier de Ribes TL, et al. Extrasynovial ultrasound abnormalities in the psoriatic finger: prospective comparative power-Doppler study versus rheumatoid arthritis. Joint Bone Spine 2006; 73:527-531.

39. Lalande Champetier de Ribes T, Margarit-Coll N, Sans N, et al. Ultrasound features of enthesopathy in patients with psoriatic dactylitis [in French]. J Radiol 2006; 87:639-645. 